\title{
Adaptive Beaconing in Mobility Aware Clustering Based MAC Protocol for Safety Message Dissemination in VANET
}

\author{
Nishu Gupta, Arun Prakash, and Rajeev Tripathi \\ Department of Electronics and Communication Engineering, Motilal Nehru National Institute of Technology Allahabad, \\ Allahabad, India \\ Correspondence should be addressed to Nishu Gupta; rel0513@mnnit.ac.in
}

Received 27 July 2016; Accepted 16 October 2016; Published 11 January 2017

Academic Editor: Oscar Esparza

Copyright (C) 2017 Nishu Gupta et al. This is an open access article distributed under the Creative Commons Attribution License, which permits unrestricted use, distribution, and reproduction in any medium, provided the original work is properly cited.

Majority of research contributions in wireless access in vehicular environment (WAVE)/IEEE 802.11p standard focus on life critical safety-related applications. These applications require regular status update of vehicle's position referred to as beaconing. Periodic beaconing in vehicle to vehicle communication leads to severe network congestion in the communication channel. The condition worsens under high vehicular density where it impacts reliability and upper bound latency of safety messages. In this paper, WAVE compliant enhancement to the existing IEEE 802.11p protocol is presented which targets prioritized delivery of safety messages while simultaneously provisioning the dissemination of nonsafety messages. Proposed scheme relies on dynamic generation of beacons to mitigate channel congestion and inefficient bandwidth utilization by reducing transmission frequency of beacons. Through the use of clustering mechanism, different beaconing frequencies and different data transmission rates are assigned to prioritize vehicular mobility. Through extensive simulation results, the performance of the proposed approach is evaluated in terms of a wide range of quality of service (QoS) parameters for two different transmission ranges. Results show that the proposed protocol provides significant enhancement and stability of the clustered topology in vehicular ad hoc network over existing standard and other protocols with similar applications.

\section{Introduction}

Vehicular ad hoc network (VANET) in recent years has emerged as a promising wireless network technology for academia, research community, and industry to support a wide range of applications ranging from travel safety to traffic management and navitainment (navigation and entertainment). VANET adopts dedicated short-range communication (DSRC) technology which is based on short-range wireless communication under the process of standardization as the wireless access in vehicular environment (WAVE)/IEEE 802.11p standard [1]. VANET facilitates wireless communication among vehicles (vehicle-to-vehicle or V2V communication) and between vehicles and road side units (vehicle-toinfrastructure or V2I Communication). V2V communication forms a basis for decentralized active safety applications that are expected to reduce accidents and their severity [2]. Safetyrelated applications emphasize avoiding the risk of road accidents. Intersection collision warning, lane merge warning, lane change alert, precrash sensing, traffic violation alert, and road condition alert are some examples. These applications have real-time constraints, timeliness being the prominent one [3]. However, the inherent features of vehicular networking such as high speed, intermittent connectivity, and frequent topological changes lead to special issues and challenges in the network design, especially at the medium access control (MAC) layer. One such issue in VANET that has yet not been addressed convincingly is how the nodes should share the radio resources in order to ensure optimum quality of service (QoS) especially for safety assurance. The safety and nonsafety message requirements are accomplished by exchanging short messages called beacons. Message dissemination through beacons in VANET is termed as beaconing. Information inside a beacon may include vehicle's location, speed, moving direction, and other driving/topographical information [4].

Since all vehicles access the same control channel, the beaconing load may eventually saturate the capacity of the 
channel. Therefore, channel congestion due to beaconing load must be reduced to avoid beacon collision and communication delay and to facilitate smooth channel access, fairness, and better reception rate. So far, several beaconing schemes [5-9] have been proposed and are classified into two main categories: periodic and adaptive. Periodic beaconing schemes broadcast beacons at regular intervals to announce the status of the vehicle to other vehicles within its vicinity [10]. The accuracy of the transmitted information depends on the beaconing rate. Higher beacon transmission rate degrades the link performance and results in messages being lost. On the contrary, low beaconing rate collects inaccurate information about neighbouring vehicles. This disparity between beaconing rate and information inaccuracy needs to be resolved. The effect of channel congestion caused by periodic beaconing can be reduced by employing adaptive beaconing schemes. Such schemes exploit the local traffic behaviour and other parameters such as vehicular density, relative speed of neighbouring vehicles, and distance between nodes to adjust beacon interval, data transmission rate, contention window (CW) size, or any combination of these. To address such issues related to channel congestion and its effects, this paper proposes to reduce the network congestion by implementing mobility based clustering incorporating adaptive beacon approach and fairly utilize the channel bandwidth and improve network reliability in message dissemination with minimum delay in the network.

(1) Contributions of the Paper. In this paper, we propose a mobility aware clustering based MAC protocol that is built on a time division multiple access (TDMA) configuration. We emphasize dissemination of safety-related applications, since they are known to have more exhaustive QoS requirements in terms of channel congestion and bandwidth utilization. We attempt to analyze how the IEEE 802.11p/1609.4 standard is affecting the performance of event-driven safety applications and in what ways can we mitigate channel congestion and improve channel utilization along with assuring reliable dissemination of safety messages in VANET environment.

The major contributions of the paper are summarized as follows:

(1) We propose mobility aware clustering based algorithm that prioritizes messages dissemination pertaining to the relative node speed.

(2) We propose an adaptive beaconing scheme to provide collision-free channel access, control congestion, and alleviate bandwidth utilization by using varying beaconing frequency and varying data transmission rates.

(3) We employ a TDMA based slot reservation technique by harnessing the multichannel feature of the WAVE standard and access the time slots on the control channel interval (CCHI) for safety messages and service channel interval (SCHI) for nonsafety messages respectively.

(4) We implement adaptive CW and backoff time mechanisms for the purveying of nonsafety messages.
(2) Organization of the Paper. The remainder of this paper is organized as follows: A brief overview of related works is presented in Section 2. Problem statement and system model are outlined in Section 3. The proposed protocol is described in Section 4. In Section 5 the performance of the proposed protocol is evaluated and simulation results are presented. Finally, conclusion and future works are presented in Section 6.

\section{Related Work}

In this section, we briefly outline the existing schemes and their methodology in the background of the proposed protocol. The discussion includes beacon congestion control method and performance parameters that are evaluated in those schemes through simulations.

The authors in [13] point out the inefficient transmission capabilities of the legacy standard. The IEEE 802.11p standard designed for wireless operations in the vehicular environment uses the carrier sense multiple access/collision avoidance (CSMA/CA) as its MAC method. However, it suffers from numerous problems. For broadcast transmission, requestto-send/clear-to-send (RTS/CTS) mechanism is infeasible because CSMA provides no means to solve the hidden station problem which can lead to a high rate of packet collision under heavy traffic load. Secondly, CSMA causes unacceptable channel access delays and therefore is unable to support real-time communication.

The multichannel procedures are majorly defined in WAVE IEEE 1609.4 standard [14] which is considered to be the default multichannel MAC standard for VANET. In a survey based on safety applications in VANET [15] the authors discuss various multichannel coordination based MAC protocols. The multichannel protocols specify MAC sublayer services and functions to support wireless connectivity among WAVE devices [16]. Figure 1 depicts the multichannel operation in IEEE 1609.4 standard. According to the type of message, they are transmitted to any of the two types of channels, either $\mathrm{CCH}$ or $\mathrm{SCH}$. The transceiver of each vehicle switches between these two channels. Each channel consists of alternating fixed length intervals, the CCHI and the SCHI whose duration of operation is fixed at $50 \mathrm{~ms}$. There lies a guard interval (GI) in between CCHI and SCHI to prevent overlapping of channels. In the $75 \mathrm{MHz}$ DSRC band, $5 \mathrm{MHz}$ bandwidth is constituted by the GI. Altogether, the sum of $\mathrm{CCH}, \mathrm{SCH}$, and $\mathrm{GI}$ form a synchronization interval (SYNC Interval) of $100 \mathrm{~ms}$ duration.

According to the coordination scheme, during the CCHI, all nodes tune to the $\mathrm{CCH}$ for exchanging safety messages and other control packets such as private service advertisements whereas during the SCHI nodes transmit nonsafety applications. As a consequence, this scheme allows the transmission of safety and nonsafety applications on different channels without missing important messages on $\mathrm{CCH}$. The best solution to provide traveler safety services and nonsafety realtime applications in order to improve driving comfort and efficiency is through the multichannel communications [15].

Whereas the use of DSRC band is not subject to any license, certain channelization mechanisms have been 


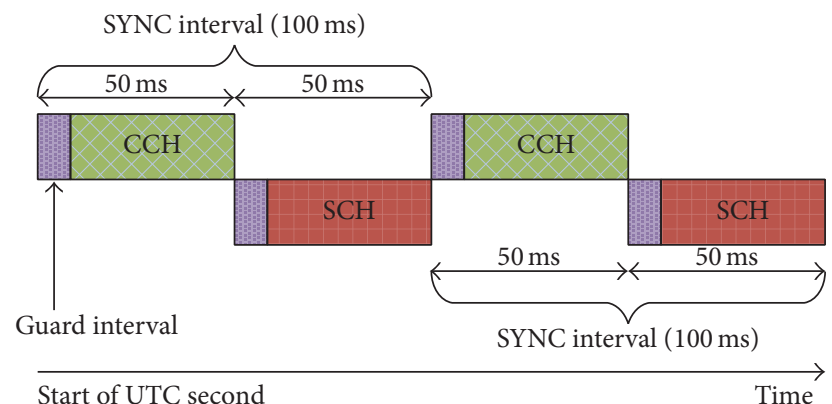

FIGURE 1: Multichannel operation in WAVE IEEE 1609.4 standard.

predefined towards its strict and effective utilization. The $75 \mathrm{MHz}$ DSRC band (5.850 to $5.925 \mathrm{GHz}$ ) is divided into seven channels of $10 \mathrm{MHz}$ numbered as $172,174,176,178$, 180, 182, and 184 as depicted in Figure 2. Channel number 178 is the $\mathrm{CCH}$. It is this channel that looks after the overall coordination between all the channels along with providing access to critical safety applications. The other six channels are SCH. SCH number 172 is reserved for High Availability and Low Latency (HALL). Channel 184 is reserved for public safety intersections. Both of these channels (172 and 184) are specifically dedicated to public safety. Channels 174 and 176 provide medium range service applications for shared public safety/private usage. SCHs 180 and 182 render short-range services for shared public safety/private usage.

In [15], the authors review a distributed and adaptive broadcasting mechanism for VANET termed as DMMAC that adopts hybrid channel access to provide collision-free and delay-bounded transmission for safety-related traffic [17]. By adopting a variable length in $\mathrm{CCH}$, DMMAC can enhance the delivery ratio of safety packets. This protocol aims to find the cluster size and hence the communication range that maintains high network stability and reliability, increases the lifetime of the path, and at the same time decreases the time delay for an emergency message to reach its intended recipients. However, the SCH resources are still wasted during the CCHI and dynamic adjustment of the $\mathrm{CCH}$ interval is not considered.

Sahoo et al. [18] propose a time-slot-based medium access protocol named as congestion-controlled-coordinator-based MAC scheme (CCC-MAC) that addresses beacons and emergency messages. In this protocol, the network is virtually partitioned into a number of segments. Within a segment, medium access is accomplished by using a time-slotscheduling mechanism supervised by a local coordinator vehicle. The proposed scheduling scheme mitigates channel congestion by reducing the transmission time of beacons through the use of multiple data rates. The paper attempts to improve bandwidth utilization by reusing the unoccupied time slots but the conventional periodic beaconing approach leads to poor channel utilization. Moreover, generating a pulse gap at the beginning of each slot further adds to channel congestion and consumes considerable bandwidth. Adding the concept of clustering along with adaptive beaconing would improve the broadcasting range in VANET environment.
With the aim of locally assigning the time slot for beaconing, a beacon scheduling scheme referred to as the context awareness beacon scheduling (CABS) is proposed in [19] which is based on spatial context information and dynamically scheduling the beacons. CABS mandates each vehicle to send packets every frame even if it does not have any message to transmit. This results in unnecessary bandwidth consumption resulting in inefficient channel utilization. In [20], dynamic clustering of vehicles within two-hop neighbourhood is discussed which provides the possibility to control resource sharing and management functions in VANET.

The existing research has been seen to be confined to beaconing techniques. They do not take mobility based clustering into consideration nor do they support reliable and efficient communication to an optimum level. Unlike the above schemes, the proposed protocol leverages the dynamic beaconing and varying data rate along with combining slot reservation mechanism in mobility based clustering of the nodes. This results in much better channel utilisation and reduced channel access delay, providing a significant enhancement over the existing IEEE 802.11p standard.

\section{Problem Statement}

3.1. System Model and Assumptions. One of the major challenges in vehicular network is dynamic topology and high node mobility. Because of these constraints, the connection between vehicles is intermittent and unpredictable. Therefore, a distributed protocol is indispensably required that relies only on local information of network instead of information of global network [4]. In the proposed system model, we focus on mobility dependent MAC mechanism where time is partitioned into frames of fixed length. We alleviate channel congestion by making beacon transmission adaptive, which is achieved by using variable data rates. The TDMA configuration is designed to counteract the interference effects induced by higher data rates. As far as the dissemination of emergency messages is concerned, the broadcasting node is rendered immediate channel access by means of a reservation mechanism. Simultaneously, we provision the dissemination of nonsafety message as well but all the time priority is given to safety message. Figure 3 shows the slot assignment scheme in SYNC interval for the broadcasting period. The process of cluster formation is performed at the beginning of a time frame. The clustered nodes communicate via single channel in purely ad hoc mode. Each node is assigned a unique ID based on its MAC address. For time coordination, all vehicles maintain synchronization with the coordinated universal time (UTC) that can be acquired from global positioning system (GPS) devices. Each node maintains a list of its one-hop neighbours. Unlike previous works $[13,17,18,21-23]$, in our approach, the nodes broadcast HELLO message (node's location, speed, moving direction, and other control information) to their one-hop neighbours dynamically and not periodically. Since the standard beaconing rate specified for vehicular safety applications is typically $100-500 \mathrm{~ms}$ [19], in the proposed beacon scheduling schemes, the beaconing interval range lies within this range. Apart from that, we impart multiple data 


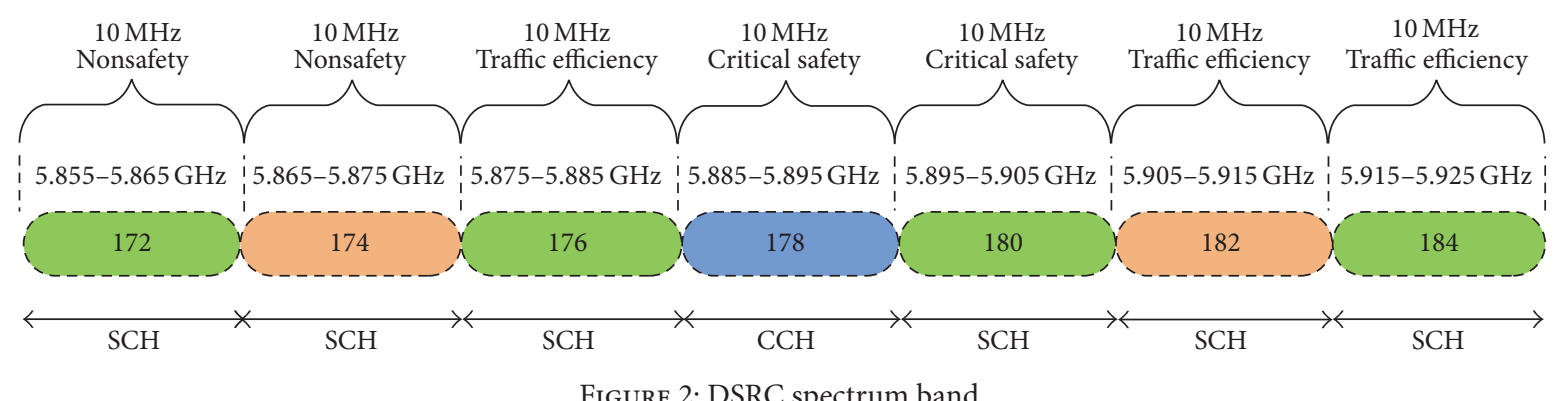

FIGURE 2: DSRC spectrum band.

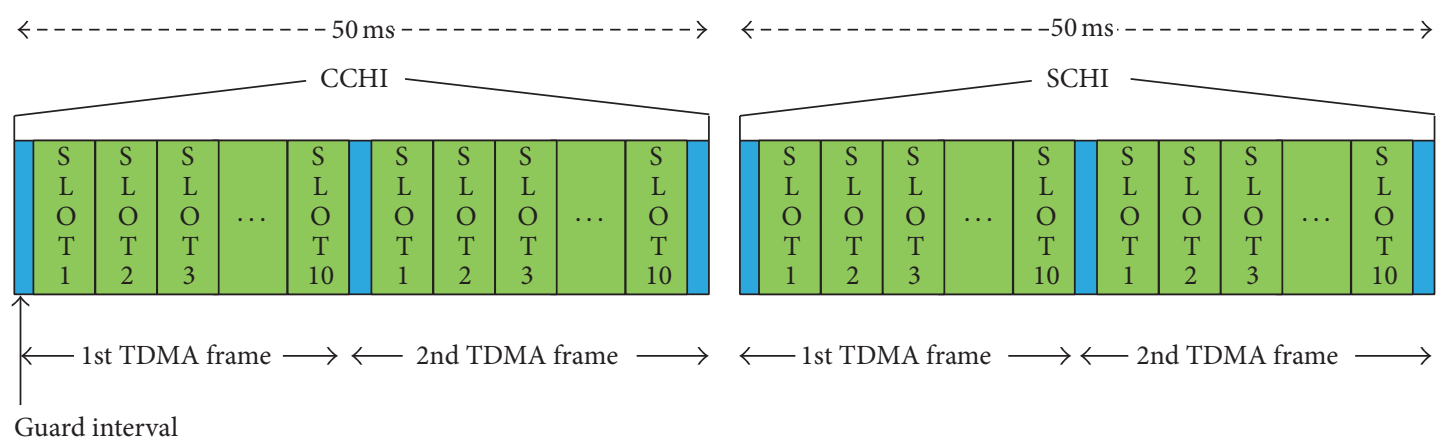

FIGURE 3: Slot assignment scheme in SYNC Interval.

rates lying in the range of 6 to $27 \mathrm{Mbps}$ which is chosen as per the prescribed range of IEEE 802.11p standard.

\section{Proposed Protocol}

In the proposed protocol, when the vehicles enter the network region for the first time, they broadcast their status message (HELLO message) independently to make other vehicles in the vicinity aware of their presence and other positional information. Once these messages are shared among the nodes in the single-hop distance network range, they form a cluster. Nodes are clustered based upon their speed. The clustering algorithm does not require additional message other than the HELLO message since the cluster formation is mobility based. Each cluster is maintained by a cluster head $(\mathrm{CH})$. A $\mathrm{CH}$ broadcasts a message that assigns the minislots in the broadcasting period to the cluster members. Every node that receives the CH's message knows its minislot and is synchronized with the other cluster members. Therefore, there are no collisions during the HELLO broadcasting period. Whenever a message is triggered at any node, it is first checked whether it is a safety message or a nonsafety message. As shown in the flow diagram (Figure 4), the proposed protocol follows different approach for each type of message. In the following subsections we discuss the mechanism adopted in the proposed work.

4.1. Safety Message Dissemination. The dissemination mechanism of high priority event-driven messages has different characteristics. They are preferred over beaconing signals. However, beaconing is equally important as it forms the basis of a diverse range of intelligent transportation systems (ITS) applications [24]. Event-driven messages are triggered only at the detection of an emergency situation and therefore are not expected to cause significant load on the channel [13]. Beacon messages, however, have a more relaxed deadline requirement and are expected to cause significant load on the channel. Especially in case of safety messages, channel availability and reliable transmission need to be ensured. The proposed protocol is so designed that it inherently prioritizes safety message by facilitating ensured channel access since it is based on TDMA-slot reservation mechanism.

4.1.1. Cluster Formation. The proposed scheme harnesses clustering based topology for its safety message dissemination process. Nodes are clustered based upon their mobility. They form dynamic clusters and the ones that are more suitably located become $\mathrm{CH}$. Selection of $\mathrm{CH}$ is the most important task in a cluster. $\mathrm{CH}$ is responsible for monitoring the data propagation process inside and between the clusters. The clusters of high-speed vehicles ensure that, even with such dynamic mobility, moving cluster architecture results in relatively stable topology as long as velocity of the vehicles remain more or less the same. Each node within a cluster is connected by one-hop intracluster link and different clusters link to each other through multihop topology.

When a vehicle enters the road for the first time, it looks for availability of any cluster by broadcasting a cluster joining request message and starts sending its status message without an elected $\mathrm{CH}$. If the vehicle receives any response, it joins the cluster whose average speed matches with its instantaneous speed. However, when the vehicle does not receive any response, it initiates the cluster formation process itself to identify cluster members by broadcasting HELLO 


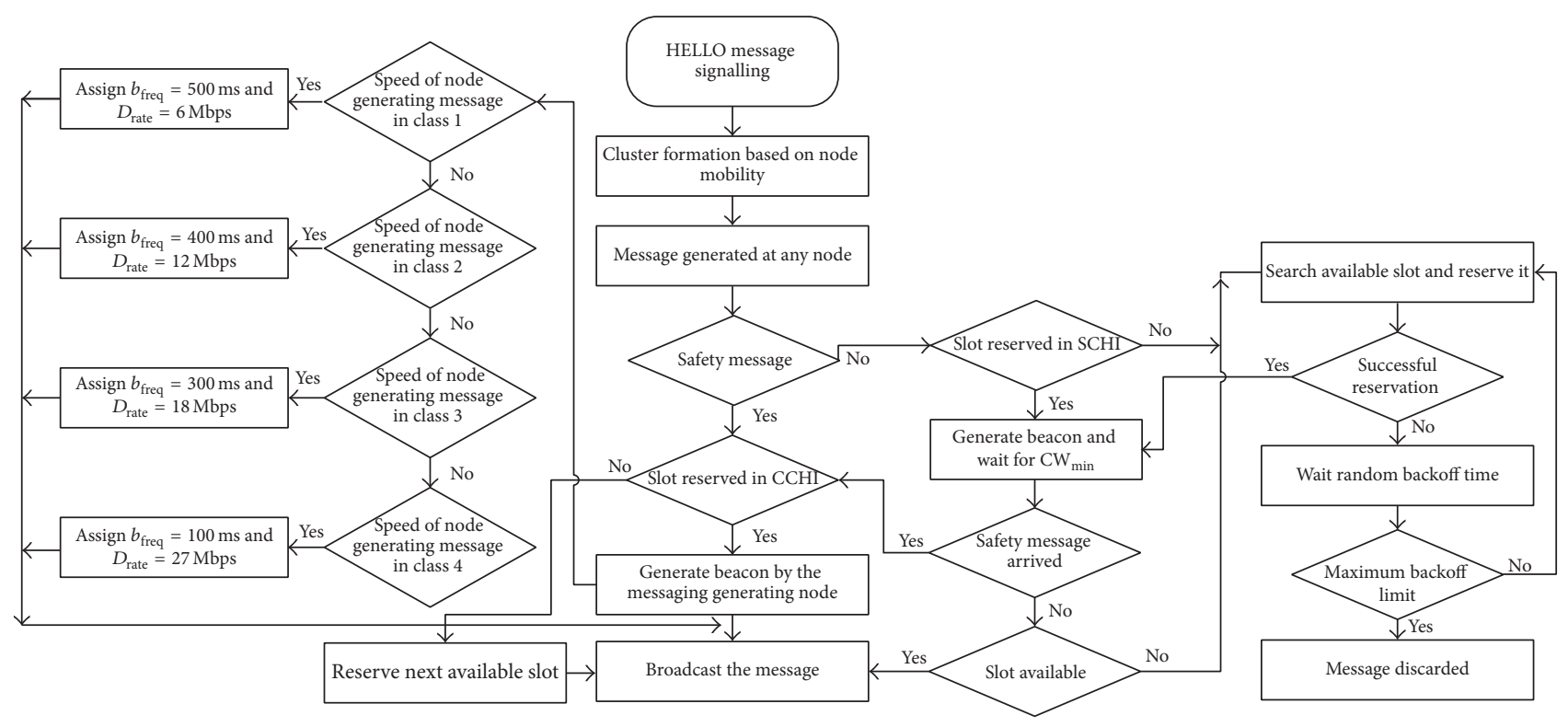

FIGURE 4: Flow diagram of the proposed protocol.

Step 1: vehicle $x$ broadcasts cluster joining request message

Step 2: if request acknowledged

Step 3: vehicle $x$ joins the cluster

Step 4: if no response

Step 5: $\quad x$ initiates cluster formation process

Step 6: end if

Step 7: end if

Step 8: vehicles $x_{1}, x_{2}, \ldots, x_{n}$ receives cluster joining request

Step 9: vehicles broadcast $T_{\text {hello }}$

Step 10: if speed of $x_{1}, x_{2}, \ldots, x_{n}<20$

Step 11: $\quad$ add $x_{1}, x_{2}, \ldots, x_{n}$ to cluster class 4

Step 12: else

Step 13: if speed $>20$ and $\leq 30$

Step 14: $\quad$ add $x_{1}, x_{2}, \ldots, x_{n}$ to cluster class 3

Step 15: else

Step 16: if speed $>30$ and $\leq 40$

Step 17: $\quad$ add $x_{1}, x_{2}, \ldots, x_{n}$ to cluster class 2

Step 18: else

Step 19: if speed $>40$

Step 20: $\quad$ add $x_{1}, x_{2}, \ldots, x_{n}$ to cluster class 1

Algorithm 1: Cluster formation process.

message $\left(T_{\text {hello }}\right)$ to other vehicles within their communication range. The vehicles moving in the same direction and in the vicinity of each other receive the request. Algorithm 1 narrates the stepwise cluster formation process.

To select a $\mathrm{CH}$, we introduce some enhancements in the scheme discussed in [15] which uses relative velocity of a vehicle to determine a weighted stabilization factor $\left(\beta_{\mathrm{WSF}}\right)$. Assuming the number of vehicles to be " $M$ " we calculate the position of different vehicles in a cluster. Let $\left(x_{1}, y_{1}\right),\left(x_{2}, y_{2}\right), \ldots,\left(x_{M}, y_{M}\right)$ be their positions. Therefore, centroid of cluster can be calculated as

$$
X=\frac{x_{1}+x_{2}+\cdots+x_{M}}{M} .
$$

Similarly,

$$
Y=\frac{y_{1}+y_{2}+\cdots+y_{M}}{M} .
$$

Distance of each vehicle from $\mathrm{CH}$ is calculated as

$$
d_{n}=\sqrt{\left(x_{n}-X\right)^{2}+\left(y_{n}-Y\right)^{2}} .
$$

Reproducing the expression in [15],

$$
\begin{aligned}
& \mathrm{CF}_{n} \\
& =\left(1-\frac{\text { distance of } n \text {th vehicle from centroid }}{\text { Transmission range }}\right),
\end{aligned}
$$

where CF is the closeness factor which includes the transmission range while selecting $\mathrm{CH}$. Value of $\mathrm{CF}$ is always less than 1. The higher is the value of $\mathrm{CF}$, the closer is the vehicle to the centroid. Cluster head coefficient $\left(\mathrm{CH}_{\text {coeff }}\right)$ establishes a relationship between $\beta_{\mathrm{WSF}}$ and $\mathrm{CF}_{j}$ as shown in

$$
\mathrm{CH}_{\text {coeff }_{n}}=\varepsilon \mathrm{CF}_{n}+(1-\varepsilon) \beta_{\mathrm{WSF}_{n}} .
$$

Value of $\varepsilon$ depends on the range and velocity of the vehicles whose value lies within $0<\varepsilon \leq 1$. Vehicle having highest $\mathrm{CH}$ coefficient is selected as $\mathrm{CH}$. For selecting cluster members with similar mobility range, first the speed range of all the nodes is compared with a threshold value based on the categories specified under different classes as specified later in Table 1 . If the speed of the node lies within the specified range 
TABLE 1: Classification of mobility range of the clusters.

\begin{tabular}{lcccc}
\hline Category & Mobility range of the node $(\mathrm{m} / \mathrm{s})$ & Beaconing interval $\left(B_{\text {intv }}\right)(\mathrm{ms})$ & Data rate $\left(D_{\text {rate }}\right)$ & Priority \\
\hline Class 1 & $40-45(\max )$ & 500 & 12 & High \\
Class 2 & $30-40$ & 400 & 18 & Medium \\
Class 3 & $20-30$ & 300 & 18 & Medium \\
Class 4 & $15-20(\mathrm{~min})$ & 100 & Low \\
\hline
\end{tabular}

Step 1: vehicle $x$ broadcasts message $P$

Step 2: another message $P^{\prime}$ is received at the same channel while $P$ is under transmission

Step 3: if priority of $P>P^{\prime}$

Step 4: $\quad$ ignore $P^{\prime}$ and broadcast $P$

Step 5: else if

priority of $P<P^{\prime}$ and

Step 6: if its current channel $=\mathrm{CCH}$

Step 7: $\quad$ broadcast $P^{\prime}$ instead of $P$

Step 8: $\quad$ broadcast $P$ after finishing broadcasting $P^{\prime}$

Step 9: else if its current channel $=\mathrm{SCH}$

Step 10: broadcast $P^{\prime}$ first and then $P$

Algorithm 2: Priority assignment mechanism for safety messages.

that node is accepted as a cluster member in the respective class. This comparison serves in assuming that the nodes are moving at relatively the same speed. This way, the cluster members in a cluster communicate with their $\mathrm{CH}$. The $\mathrm{CH}$ can communicate beyond the cluster boundaries using the $\mathrm{CCH}$ and send the status information to the next $\mathrm{CH}$ node and likewise multihop transmission is accomplished.

4.1.2. Slot Reservation Mechanism. We leverage the multichannel feature of the WAVE standard to implement the slot reservation mechanism in the proposed protocol. $\mathrm{CCH}$ and $\mathrm{SCH}$ are partitioned into two frames of equal duration. These frames are further divided into a number of slots (10 here). Safety messages can be delivered on any of the two channels $(\mathrm{CCH}$ and $\mathrm{SCH})$ whereas nonsafety messages are assigned $\mathrm{SCH}$ for message dissemination. However, if none of the slots are available in the $\mathrm{CCH}$ for safety messages, the nodes can reserve the next available slots and broadcast their message. Algorithm 2 demonstrates the mechanism through which safety messages are prioritized over nonsafety messages.

The slot reservation phase consists of the time allocated for intracluster and intercluster communication. In intracluster communication phase when a node encounters a safety message, it raises a beacon message informing the $\mathrm{CH}$ about message arrival. Each cluster member will receive a different time slot for sending its beacon to its $\mathrm{CH}$. In case of intercluster communication, to ensure reliable message transmission with minimal delay, every node is assigned a slot in the TDMA frame during the CCHI SCHI to transmit its message. Depending upon the mobility range of the cluster to which the message carrying node belongs, a predefined beaconing frequency and data rate are assigned which is discussed in detail in the next subsection. In order to deliver an event-driven message, the node first checks if there is any reserved time slot. If a slot is found reserved, the node transmits its message and sends it to the lower layer. Otherwise, the node reserves the next available time slot for itself and uses it for message transmission. Figure 5 depicts the above discussed clustering based slot assignment process.

The proposed slot-based reservation mechanism specifically designed for safety message dissemination guarantees reliability as the emergency message is transmitted in reserved time slots, ensuring collision-free transmission.

Now, we calculate the time required for message delivery phase and slot assignment phase through analytical means. The delivery phase duration is calculated as

$$
T_{\text {DEL }}=M_{R}\left(\frac{M_{\text {size }}}{D_{\text {rate }}}+T_{\text {hello }}\right),
$$

where $M_{R}$ is the number of mobility ranges (four in the proposed work), $M_{\text {size }}$ is size of the $\mathrm{CH}$ message, $D_{\text {rate }}$ is the data rate assigned to the node, and $T_{\text {hello }}$ is time duration required in transmitting the message header.

The beaconing message consisting of various pieces of positional information of the vehicle can be expressed as

$$
M_{\text {beacon }}=S_{\mathrm{CM}}+S_{\mathrm{OH}},
$$

where $S_{\mathrm{CM}}$ is payload size of the message carrying node and $S_{\mathrm{OH}}$ is the size of the associated overhead.

In slot assignment phase, nodes from adjacent clusters are scheduled to access the channel at different time slots. This is achieved by exploiting the mobility aware clustering scheme which assigns different priority levels to the clusters. Accordingly, the slot assignment phase duration is given as

$$
T_{\mathrm{SA}}=M_{R}\left(\frac{M_{\text {size }}}{D_{\text {rate }}}+T_{\text {hello }}\right)+M_{R} * X,
$$

where

$$
X=D_{\text {rate }} * T_{\text {slot }}
$$

and $T_{\text {slot }}$ in IEEE $802.11 \mathrm{p}$ is fixed at $13 \mu \mathrm{s}$.

By adding (6) and (8), we deduce the overall time required for safety message dissemination as

$$
\begin{aligned}
T_{\text {overall }} & =T_{\mathrm{DEL}}+T_{\mathrm{SA}} \\
& =2\left[M_{R}\left(\frac{M_{\text {size }}}{D_{\text {rate }}}+T_{\text {hello }}\right)\right]+\left(M_{R} * X\right) .
\end{aligned}
$$




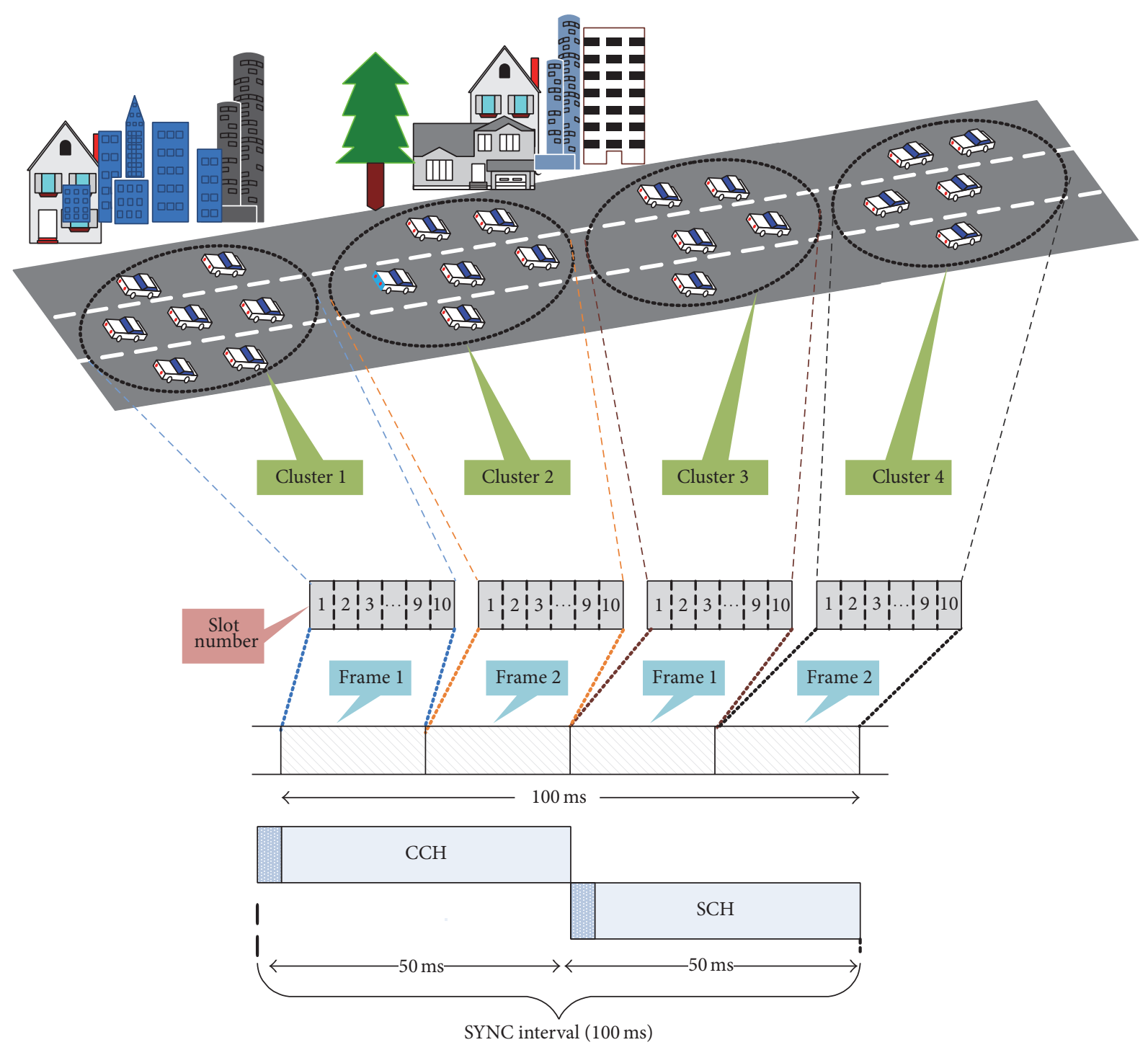

FIGURE 5: Clustering based slot assignment mechanism in multichannel scenario.

4.1.3. Adaptive Beaconing Frequency and Data Rate Generation Mechanism. For the mobility adaptive MAC, the mobility range, beaconing interval, data rate, and the respective channel access priorities are given in Table 1 . We have classified the clusters into four different classes based on their mobility range with the assumption that vehicles are travelling in the same direction (one way). Therefore, all neighbouring nodes are limited to only those vehicles which are travelling in the same direction. Vehicles having their speed within $40-45 \mathrm{~m} / \mathrm{s}$ are clustered into one cluster. Vehicles in the speed range of $30-40 \mathrm{~m} / \mathrm{s}$ are partitioned into another cluster. Likewise, based on different speed range vehicles are partitioned into different clusters. This clustering scheme considers the degree of the speed difference among neighbouring nodes as a parameter to develop stable clustering structure. A cluster having longer travel time has lower eligibility value to access the channel. Hence it is assigned lower priority (Class 4 ) as compared to other clusters. Similarly, cluster having highest node mobility is given highest priority (Class 1) as it will get least time to access the channel and broadcast its message. As we prioritize the clusters, we vary the assigned data rate and beaconing frequency as shown. Moreover, for precise information update, beacons must be generated within 100-500 ms [25]. Without proper coordination at the MAC layer, beacon generation at this rate would result in channel congestion in regions of high vehicular density, thereby causing serious degradation in the QoS performance of the network [18].

Algorithm 3 describes the beaconing interval $\left(B_{\text {intv }}\right)$ and data rate $\left(D_{\text {rate }}\right)$ assignment process based on the adopted system model. Extending our discussion in Section 3 in relation to the beaconing interval and data rate, we have assigned $6 \mathrm{Mbps}$ transfer rate to Class 1 clusters. We cite our previous work [26] behind this selection which shows that optimum values of QoS performance parameters are achieved at around $6 \mathrm{Mbps}$. That means a cluster with maximum speed range, getting least channel access time, should be assigned such a beaconing frequency and data rate that ensures high 


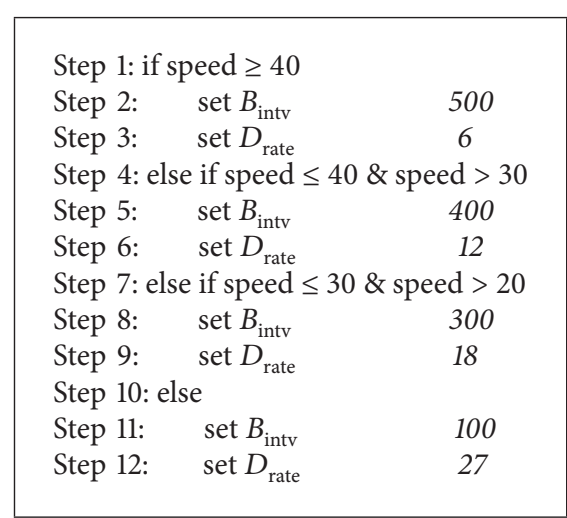

Algorithm 3: Assigning beaconing interval and data rate.

packet delivery ratio (PDR), high throughput, and low delay which is accomplished in the proposed scenario using $6 \mathrm{Mbps}$ data rate.

4.2. Nonsafety Message Dissemination. As far as nonsafety message dissemination is concerned, frame length is essential in order to improve the performance of data throughput and frame loss rate in communication network as specified in [27]. For delivering nonsafety messages, one-sixth of the total bandwidth in the SCH interval is used if only one of the SCHs is used [28]. The authors in [29] by analytical means calculate the maximum CW size. They rely upon the relative distance and SNR values between the communicating entities.

4.2.1. Modelling Backoff Interval and Access Delay for Nonsafety Messages. Standard wireless local area network (WLAN) protocol uses binary exponential backoff interval scheme whereas IEEE 802.11p standard employs $p$-persistent CSMA/CA protocol where the backoff interval is based on a geometric distribution with a specific probability of transmission, $p$. The memoryless backoff property of $p$ persistent CSMA/CA makes it suitable for the purpose of analytical modelling.

Based on this backoff interval scheme, the probability density function of having a successful transmission after $x$ failures is analyzed as

$$
P(X=x)=q^{x-1} p ; \quad x=1,2,3, \ldots
$$

and cumulative density function is given as

$$
\begin{aligned}
P(X \leq x) & =P(X=1)+P(X=2)+\cdots+P(X=x) \\
& =p+q p+q^{2} p+\cdots+q^{x-1} p=p \frac{\left[1-q^{x}\right]}{(1-q)} \\
& =1-q^{x}
\end{aligned}
$$

where $X$ is the geometric random variable or the total number of trials made for a successful transmission, $p$ is the probability of successful transmission, and $q$ is the probability of unsuccessful transmission having value equal to $1-p$. Enhancing the analytical approach adopted in $[3,30,31]$, we have applied a similar logic to calculate the expected value of $X$ denoted as $E[X]$ and how can this value be used to determine the CW is analyzed below.

$$
\begin{aligned}
& E[X]=\sum_{x=1}^{\infty} x(q)^{x-1} p=\frac{1}{p}, \\
& E[X]=\frac{\mathrm{CW}}{2}+1
\end{aligned}
$$

and from (13),

$$
\frac{\mathrm{CW}}{2}+1=\frac{1}{p}
$$

The distributed coordination function (DCF) mechanism of IEEE 802.11 WLAN dictates that a node willing to transmit would first sense the channel to be idle for arbitration interframe space (AIFS) and determine random backoff time, $T_{B}$. This backoff time is a random number that normally depends upon the number of attempted unsuccessful transmissions. To calculate $T_{B}$, for each retransmission a value from the uniformly distributed interval in the range of $[0, \mathrm{CW}]$ is randomly chosen and multiplied by the average time required to send out a frame. Initially, $\mathrm{CW}$ is set to $\mathrm{CW}_{\min }$. With passing time, different relay nodes count down each $T_{B}$. When the first node counts down to zero and detect an idle channel, it broadcasts its message. The node having smallest $T_{B}$ compared to others will attain the highest priority to broadcast. That is, the duration of $T_{B}$ decides the sequence of broadcasting among the nodes.

While modelling MAC access delay, we assume that the collision resolution time is included in the backoff period, like in [32], with a mean duration of $1 / \gamma$, which denotes the mean delay per retry. The backoff mechanism in WAVE prescribes the backoff period $\gamma$. However, if the subsequent transmission attempt fails, the interval is doubled by increasing the $\mathrm{CW}$ value until it reaches $\mathrm{CW}_{\max }$. Different AIFS number (AIFSN) and CW values are selected for different types of access categories (ACs) in the $\mathrm{CCH}$ and SCH interval. Table 2 shows different contention parameters used on the $\mathrm{CCH}$ and SCH intervals of IEEE 802.11p for different ACs along with the AIFS duration calculated from [1].

The waiting time $T_{\text {AIFS }}$ for an $\mathrm{AC}$ is calculated as

$$
T_{\mathrm{AIFS}}=\mathrm{SIFS}+\mathrm{AIFSN}_{\mathrm{AC}} * t_{\text {slot }}
$$

where SIFS (Short Interframe Space) is $32 \mu \mathrm{s}$ and $t_{\text {slot }}$ (time slot) is $13 \mu$ s for the IEEE 802.11p PHY layer with orthogonal frequency division multiplexing (OFDM) $10 \mathrm{MHz}$ as defined in [1].

And backoff period is given as

$$
\gamma=\frac{1}{T_{\mathrm{AIFS}}+\mathrm{CW}_{\min }\left(t_{\text {slot }}\right)} .
$$

Accordingly, the backoff period for each AC is given as

$$
\gamma_{[\mathrm{AC}]}=\frac{1}{T_{\mathrm{AIFS}[\mathrm{AC}]}+\mathrm{CW}_{\min [\mathrm{AC}]}\left(t_{\text {slot }}\right)} .
$$


TABLE 2: Contention parameters for CCH interval and SCH interval in IEEE 802.11p [10-12].

\begin{tabular}{|c|c|c|c|c|c|c|c|c|c|}
\hline \multirow{2}{*}{ AC number } & \multirow{2}{*}{ Access category } & \multicolumn{4}{|c|}{$\mathrm{CCH}$ interval } & \multicolumn{4}{|c|}{ SCH interval } \\
\hline & & $\mathrm{CW}_{\min }$ & $\mathrm{CW}_{\max }$ & AIFSN & $T_{\mathrm{AIFS}}(\mu \mathrm{s})$ & $\mathrm{CW}_{\min }$ & $\mathrm{CW}_{\max }$ & AIFSN & $T_{\mathrm{AIFS}}(\mu \mathrm{s})$ \\
\hline 0 & Background traffic (BK) & 15 & 1023 & 9 & 149 & 31 & 1023 & 7 & 123 \\
\hline 1 & Best effort (BE) & 7 & 15 & 6 & 110 & 31 & 1023 & 3 & 71 \\
\hline 2 & Video (VI) & 3 & 7 & 3 & 71 & 15 & 31 & 2 & 58 \\
\hline 3 & Voice (VO) & 3 & 7 & 2 & 58 & 7 & 15 & 2 & 58 \\
\hline
\end{tabular}

For the sake of clarity to the researchers, it is noteworthy that in several studies there is confusion about the value of $\mathrm{CW}_{\max }$. Based on the IEEE 802.11p standard and for the OFDM PHY layer with $10 \mathrm{MHz}$, the value of $\mathrm{CW}_{\max }$ is 1023 whereas the IEEE 1609.4 specification standard indicates the value of $\mathrm{CW}_{\max }$ to be 511 .

\section{Performance Evaluation}

The aim of this simulation study is to evaluate the V2V communication performance aspect of safety as well as nonsafety applications where all the vehicles are equipped with on-board unit enabling wireless communication. Vehicles communicate with each other in a clustered scenario to collectively exchange data with neighbouring vehicles. Simulation results and their evaluation are used to compare the performance of different protocols for QoS metrics of throughput, PDR, end-to-end delay, and packet loss ratio along with some clustering related parameters such as average $\mathrm{CH}$ time, emergency message interarrival time, and protocol overhead. We have evaluated different protocols for two different transmission ranges, $300 \mathrm{~m}$ and $500 \mathrm{~m}$, owing to the existing literatures suggesting the optimum V2V communication range to be around $300 \mathrm{~m}$. However, some researchers have opted for a higher communication range also. So, to have a discreet and in-depth analysis of the comparison and identify better communication range in V2V scenario, we have extended our simulation to $500 \mathrm{~m}$ as well.

5.1. Experimental Setup. In order to evaluate the performance of the proposed protocol, simulations are performed using ns-2 simulator [33] version ns-2.34, which is modelled to provide DSRC MAC layer specifications. A total of 100 nodes are deployed in a region of size $10000 \mathrm{~m} \times 10000 \mathrm{~m}$. IEEE 802.11 DCF is selected as MAC layer protocol. The MAC layer data rate is set to vary within $6-27 \mathrm{Mbps}$ depending upon the node mobility. We consider varying node speed in the range of $15-45 \mathrm{~m} / \mathrm{s}(54 \mathrm{~km} / \mathrm{h}-162 \mathrm{~km} / \mathrm{h})$. The simulation time is $300 \mathrm{sec}$. Following the recommended beaconing frequency for the vehicular safety applications, in the proposed scheme the beaconing frequency is set to vary within $2-10 \mathrm{~Hz}(100-$ $500 \mathrm{~ms}$ ) [34] depending upon the mobility of the node. Additionally, in order to perform the simulation in reliable communication environment, Nakagami- $m$ fading model is used as the signal propagation model for the vehicle communication in a highway environment. We consider a highway scenario as shown in Figure 6 where vehicles are placed on a six-lane highway with three lanes in each direction. We used a realistic mobility model generator built on the simulator

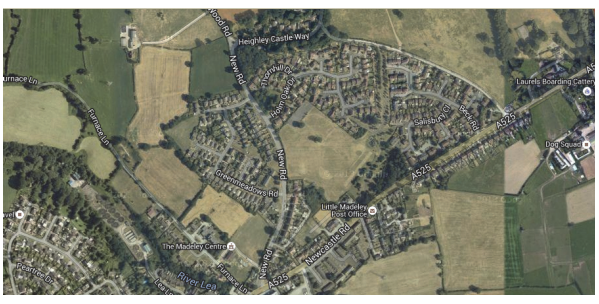

FIGURE 6: A snap shot of the highway scenario under simulation.

TABLE 3: Simulation parameters.

\begin{tabular}{lc}
\hline Parameter & Value \\
\hline Simulation map & $10000 \times 10000 \mathrm{~m}$ \\
Simulation time & $300 \mathrm{sec}$ \\
Vehicle speed & $15-45 \mathrm{~m} / \mathrm{s}$ \\
Number of vehicles & Maximum 100 \\
Number of lanes & 6 (3 in each direction) \\
Scenario & Two-way highway \\
Network interface & Phy/WirelessPhyEt \\
MAC interface & Mac/802_11Ext \\
Interface queue & Queue/DSRC \\
Propagation model & Propagation/Nakagami \\
Number of TDMA slots/frames & 10 \\
Time slot & $2.5 \mathrm{~ms}$ \\
Message size (safety) & 100 bytes \\
Message size (nonsafety) & 512 bytes \\
Transmission range & $300 \mathrm{~m}, 500 \mathrm{~m}$ \\
Modulation type & BPSK \\
Antenna type & Antenna/omniantenna \\
Channel type & Channel/wireless channel \\
Data transfer rate & $6,12,18,27$ Mbps \\
Minimum beaconing interval & $100 \mathrm{~ms}$ \\
Maximum beaconing interval & $500 \mathrm{~ms}$ \\
\hline
\end{tabular}

SUMO [35] to produce vehicular mobility traces, in which vehicles derive speeds in the range of $15-45 \mathrm{~m} / \mathrm{s}$. Other simulation parameters are listed in Table 3.

5.2. Protocols Compared. A comprehensive simulation was conducted to evaluate the performance of the proposed MAC scheme. We compare the results of the proposed protocol with the DCF of IEEE 802.11p [1], DMMAC [17], and D-FPAV [36] since these protocols carry maximum relevance to the proposed work in respect to comparability. We specify $300 \mathrm{~m}$ and $500 \mathrm{~m}$ transmission range for event-driven messages. We 
distinguish the results of the compared protocols based upon these two different ranges which strengthens the evaluation and provides significant conclusions. To enhance accuracy in the simulation work, we adopted a confidence interval range of $95 \%$ and for each case we replicated the simulation runs 5 times.

5.3. Results. Figures 7-9 show the impact of vehicle density on the QoS parameters for two different communication ranges. Figure 7 (a) compares the throughputs for $300 \mathrm{~m}$ transmission range. It shows that the proposed protocol outperforms other compared schemes. Whereas the performance of DMMAC is better than IEEE 802.11p and $\mathrm{D}-\mathrm{FPAV}$, it is much below the proposed protocol. This is because the proposed scheme alleviates message delivery rate by incorporating a mobility based clustering scenario. Similar results were obtained for $500 \mathrm{~m}$ transmission range as shown in Figure 7(b). The throughput value for this range is on a little higher side as compared to $300 \mathrm{~m}$ range.

Figures $8(\mathrm{a})$ and 8 (b) show the increase in the PDR with respect to the increase in node density for $300 \mathrm{~m}$ and $500 \mathrm{~m}$ transmission range. The PDR reaches value near about 0.9 (equivalent to $90 \%$ ) as the vehicular density approaches 100 nodes for the proposed protocol. However, for DMMAC, the maximum PDR attained is 0.36 for both the transmission ranges when the number of vehicles reaches 100 . In the proposed protocol, any or all of the members can reserve the slot and send their message. Apparently, the delivery ratio would definitely increase by increasing the number of vehicles in a given area since there will be more number of reserved nodes to transmit and eventually receive. This explains why the proposed protocol has better performance than other compared schemes particularly under high vehicular density. The other two protocols D-FPAV and IEEE 802.11p fail to deliver comparable results with their PDR lying in the range of 0.12 to 0.29 .

Figures 9(a) and 9(b) compare the end-to-end delay of the proposed protocol with those of other protocols under evaluation. The delay of event-driven safety messages for the IEEE 802.11p protocol is higher than the rest of the protocols for all range of vehicular density. For D-FPAV protocol, the delay limit is below $100 \mathrm{~ms}$ for high vehicular density but for sparse scenario, this value surpasses the delay bound limit of $100 \mathrm{~ms}$ required for most of the safety applications [37]. DMMAC protocol performs much better than the previously discussed protocols and confines its delay limit much below $100 \mathrm{~ms}$ for all vehicular densities and transmission ranges. At the lowest node density, it shows a delay of $31 \mathrm{~ms}$ and $32 \mathrm{~ms}$ for highest node density at a transmission range of $500 \mathrm{~m}$. However, the proposed protocol outperforms all other protocols with minimum value of delay caused although it is marginally lower DMMAC. This outperforming of the proposed protocol is due to its ability to reduce the probability of transmission collision as compared to DMMAC, D-FPAV, and IEEE 802.11p protocol.

Next, in Figures 10(a) and 10(b), we evaluate the emergency message interarrival time, which is an important parameter in determining the efficiency of beacon-based safety applications. Ideally, it lies in the range of recommended beaconing frequency interval for vehicular safety applications (i.e., $2-10 \mathrm{~Hz}$ ). It usually increases when the transmitted beacons fail to reach their destination. As shown in Figure 10, the interarrival times of the proposed protocol and DMMAC protocol overlap each other. It can be seen from the figures that as the number of vehicles increases, the interarrival time decreases. This is pretty obvious and self-explanatory. For 25 vehicles the emergency message interarrival time is around $33 \mathrm{~ms}$ which decreases to a value of $17 \mathrm{~ms}$ for 100 vehicles. However, in $802.11 \mathrm{p}$ beacons can easily surpass $150 \mathrm{~ms}$ even under vehicle density of around 100 vehicles. For the worse, it experiences more than $500 \mathrm{~ms}$ at low density (25 vehicles). The rationale behind such deterioration is that as density increases, reception rate decreases and as beacons are lost, the time gap is increased. In addition to this fact, one more reason is that beacons get obsolete and dropped due to large contention delays in IEEE 802.11p. On the other hand, DFPAV is seen to achieve comparatively lower interarrival times than IEEE 802.11p.

Figures 11(a) and 11(b) analyze the packet loss ratio and show that it decreases as the node density increases. This is pretty normal behaviour of the network as more transmitting nodes lead to more deliveries of packets and eventually low packet loss. The packet loss ratio of the proposed protocol is seen to be the least among all protocols attributing to the slot reservation mechanism which ensures reliable dissemination of message. This ratio falls to a value of 0.09 as compared to DMMAC where this ratio is 0.63 when the node density approaches 100 .

Figure 12 shows that the CH's average lifetime increases with increasing vehicular density for different transmission ranges. This is because the stability of the cluster and the $\mathrm{CH}$ increases when the number of nodes is high, owing to the reduced distance between them. It can be seen that the $\mathrm{CH}$ durations for the proposed protocol and DMMAC are the same and overlap whereas the $\mathrm{CH}$ duration is lower for DFPAV and IEEE 802.11p protocol. Moreover, this parameter shows better results for $500 \mathrm{~m}$ transmission range compared to $300 \mathrm{~m}$.

Figure 13 shows the cluster management overhead when the communication range is $300 \mathrm{~m}$ as a function of vehicle density for the proposed protocol and DMMAC. It is obvious that as the vehicle density increases, the overhead percentage decreases since more vehicles manage to send their status messages. The overhead for the proposed protocol is much lower than that of DMMAC since the $\mathrm{CH}$ in DMMAC has an additional role of selecting a backup $\mathrm{CH}$ if it has a higher stability factor than the current $\mathrm{CH}$. Whereas this increases the cluster stability, it poses additional overhead as well which is not the case in the proposed protocol.

From the above analysis, it can be observed that the proposed protocol surmounts the performance of DMMAC, D-FPAV, and IEEE 802.11p. The slot reservation scheme integrated with mobility aware clustering technique helps in establishing a reliable network as compared to other protocols. The algorithm for priority assignment to safety messages allows faster and real time delivery of event-driven life critical message dissemination. 


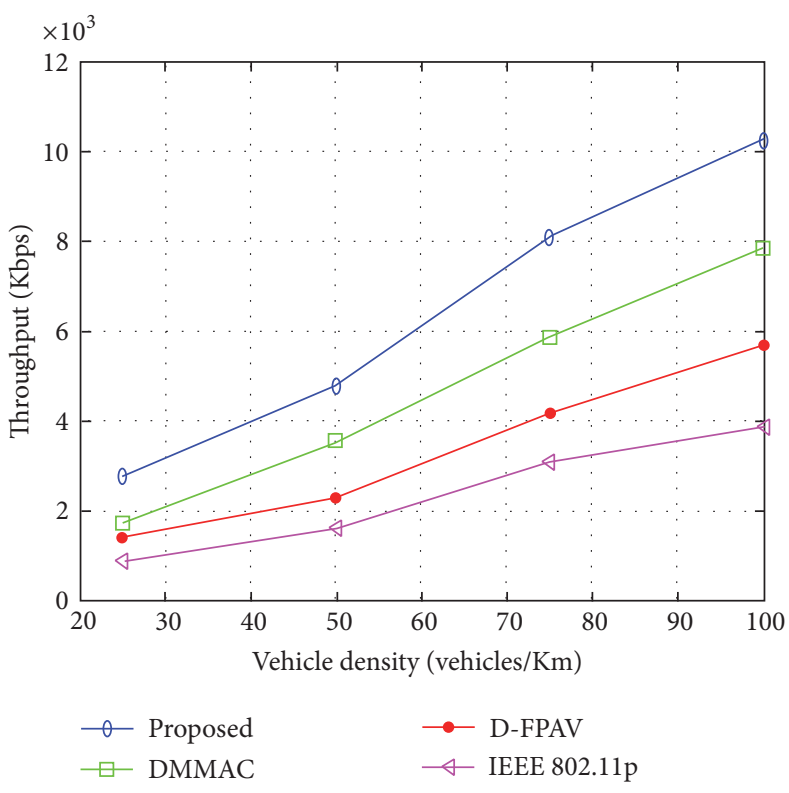

(a) $R=300$

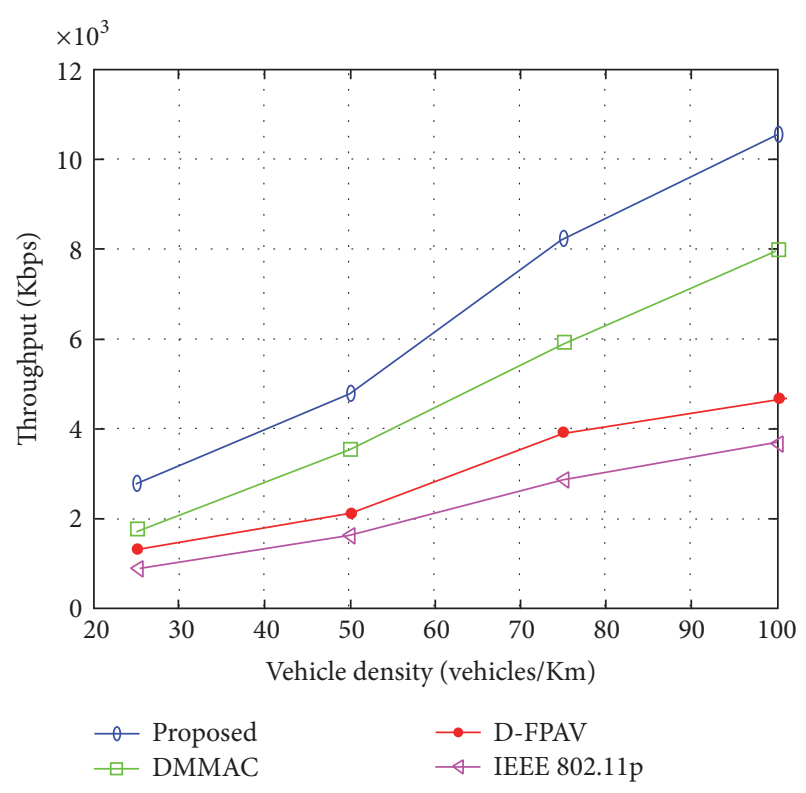

(b) $R=500$

FIGURE 7: Comparison between different protocols with respect to vehicle density and throughput for (a) $300 \mathrm{~m}$ and (b) $500 \mathrm{~m}$ transmission range.

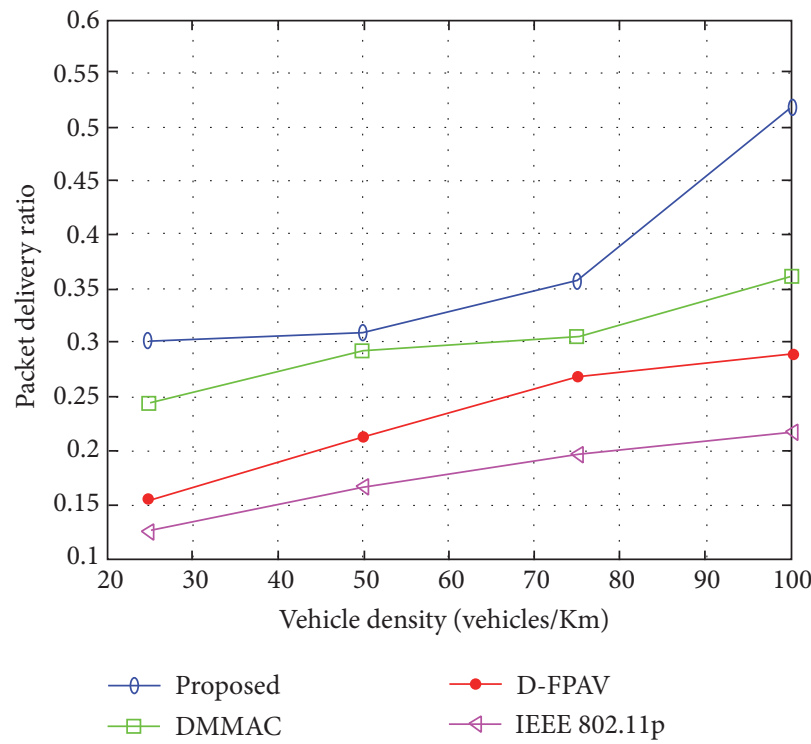

(a) $R=300$

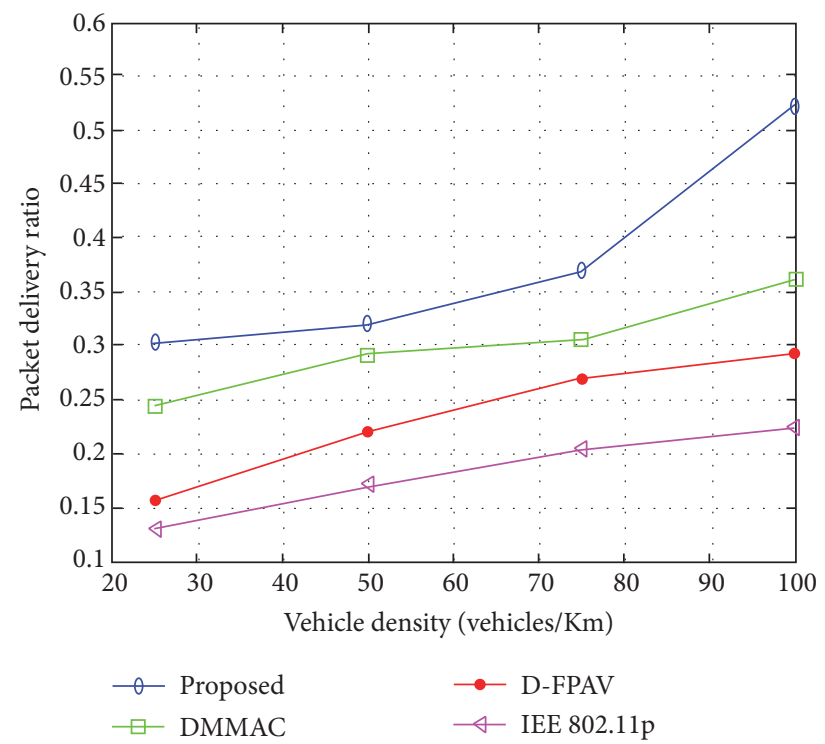

(b) $R=500$

FIGURE 8: PDR as a function of vehicle density for (a) $R=300 \mathrm{~m}$ and (b) $500 \mathrm{~m}$.

\section{Conclusion and Future Work}

In this paper, a safety oriented MAC protocol for VANET has been proposed which addresses delivery of safety messages in an efficient and reliable manner. The protocol incorporates mobility based clustering and slot reservation mechanism to reduce congestion and facilitate faster dissemination. $\mathrm{CH}$ are elected in a distributed manner according to their relative speed and distance from cluster members. The $\mathrm{CH}$ exhibits long average lifetime. Status messages are exchanged within a cluster following a sequence advertised by the $\mathrm{CH}$. The multichannel feature of WAVE is harnessed while assigning the slots to the nodes. We advocate the use of multiple data rates to make the protocol scalable and avoid congestion in the presence of significant vehicle density. Then, vehicles are provided time slots in the transmission period of their respective frames.

Upon comparing the obtained simulation results with the IEEE 802.11p standard and some multichannel clustering protocols, it is clear that the proposed protocol has high 


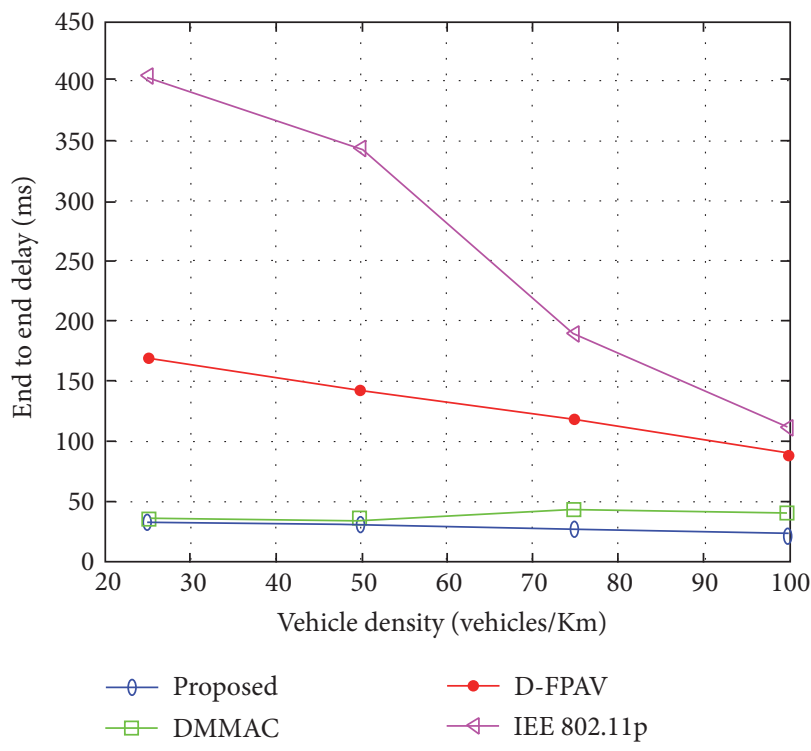

(a) $R=300$

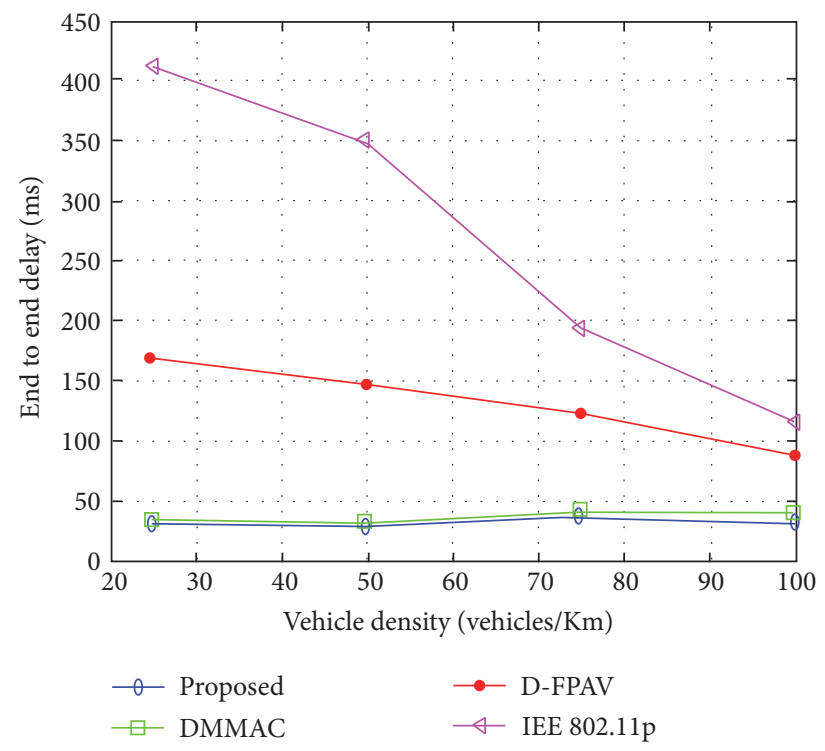

(b) $R=500$

Figure 9: End-to-end delay for transmission range of (a) $300 \mathrm{~m}$ and (b) $500 \mathrm{~m}$.

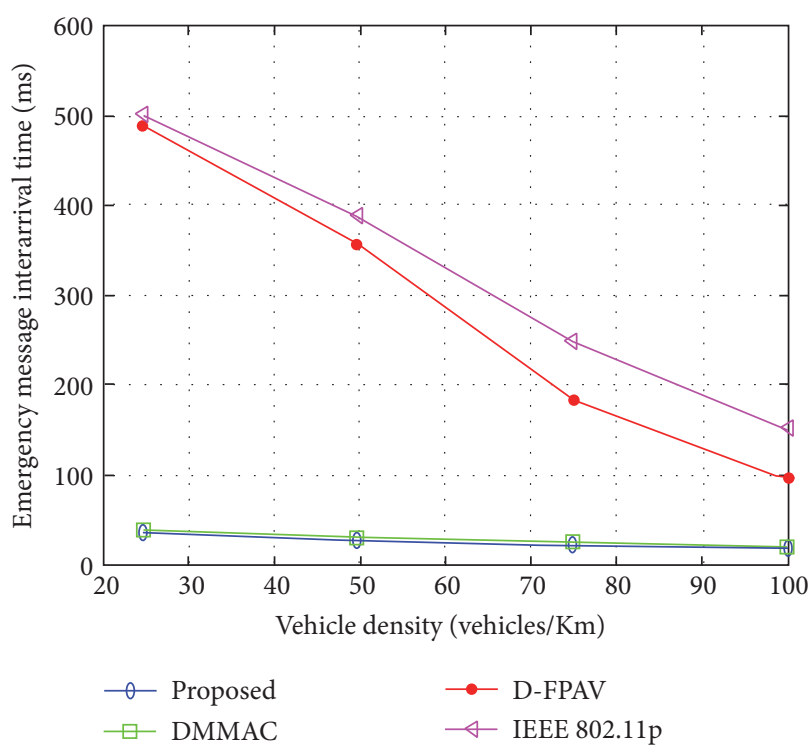

(a) $R=300$

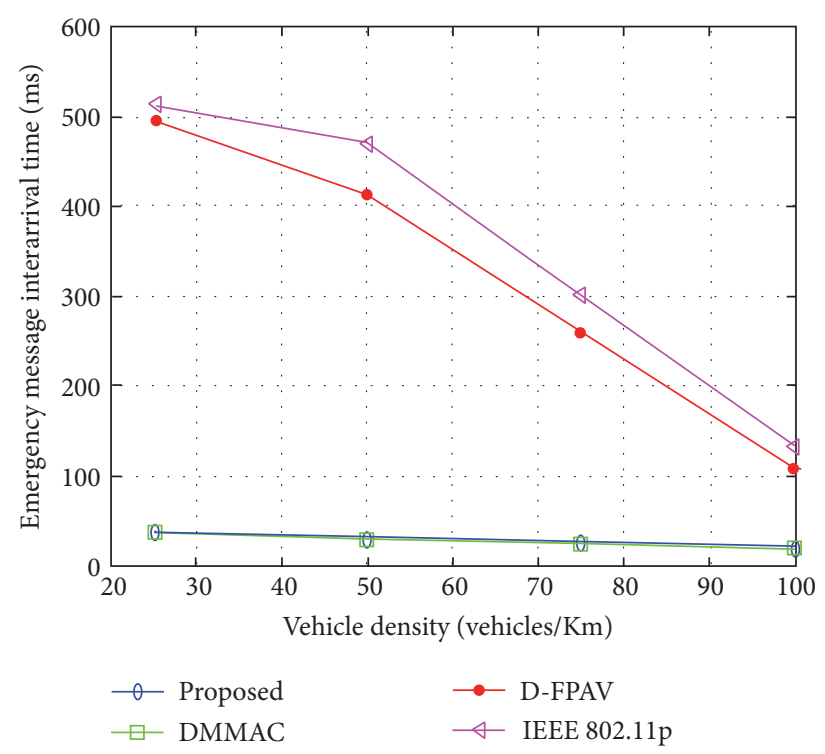

(b) $R=500$

FIGURE 10: Emergency message interarrival time for transmission range of (a) $300 \mathrm{~m}$ and (b) $500 \mathrm{~m}$.

stability and its performance exceeds other protocols. Moreover, it can achieve timely and reliable delivery of emergency messages. In addition, the protocol provides significant improvement in latency values under all density conditions. The proposed methodology provides a practical, efficient, and reliable approach to gain substantial congestion control in vehicular environment. However, further research with respect to the specific requirements of safety applications in different real-time scenarios is required to realize the potential this technology brings in to the vehicular networking environment. The proposed protocol can be further extended to work upon factors like adaptive transmission power rate and channel fading effects to alleviate the effects of SINR. In the light of the discussed protocol, we highlight below future research scope in this field.

(1) Further research is required in investigating the performance of the adaptive schemes with respect to more than one mobility factor and designing the corresponding MAC protocol.

(2) Simultaneous implementation of both safety and nonsafety applications without compromising communication of any application is another challenging issue in VANET. This needs to be investigated further since 


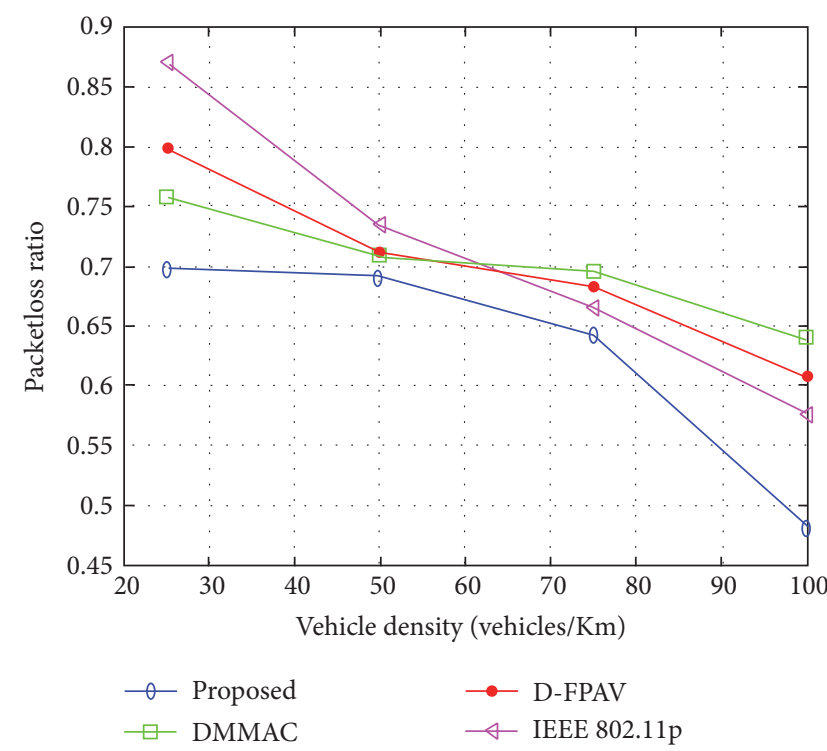

(a) $R=300$

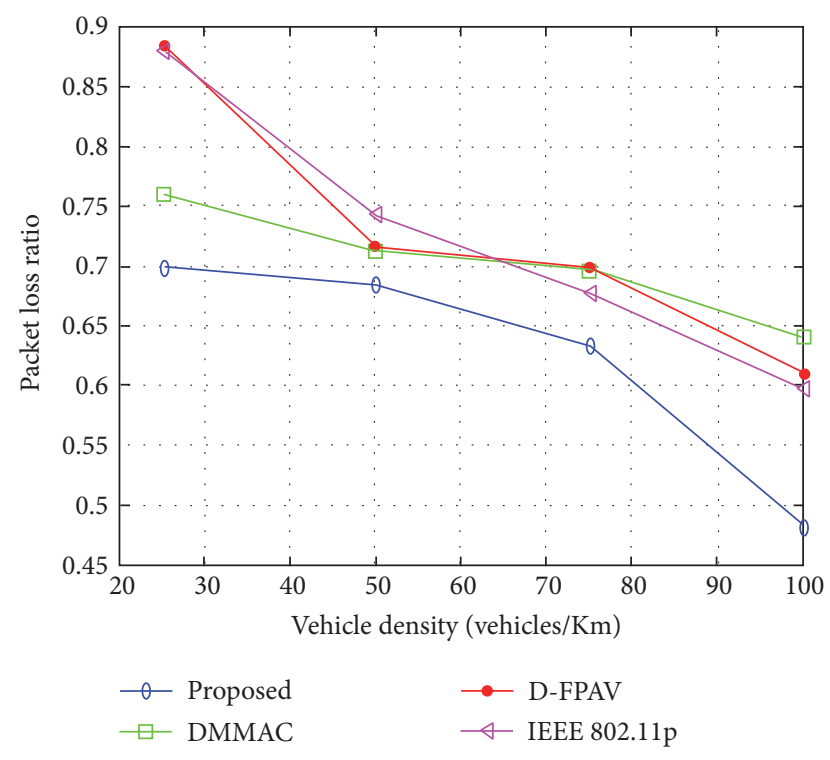

(b) $R=500$

FIGURE 11: Packet loss ratio versus vehicle density for (a) $300 \mathrm{~m}$ and (b) $500 \mathrm{~m}$ transmission range.

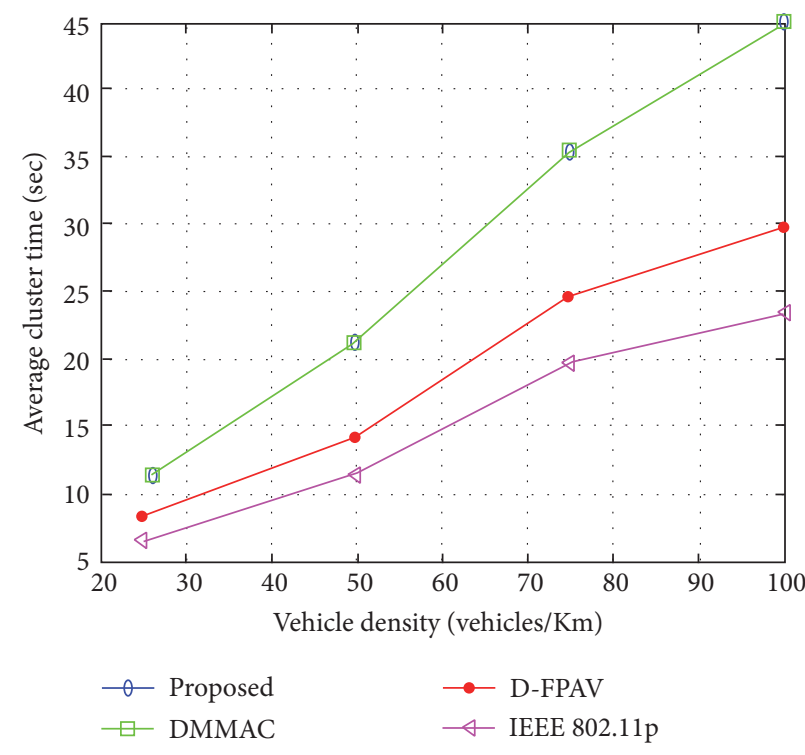

(a) $R=300$

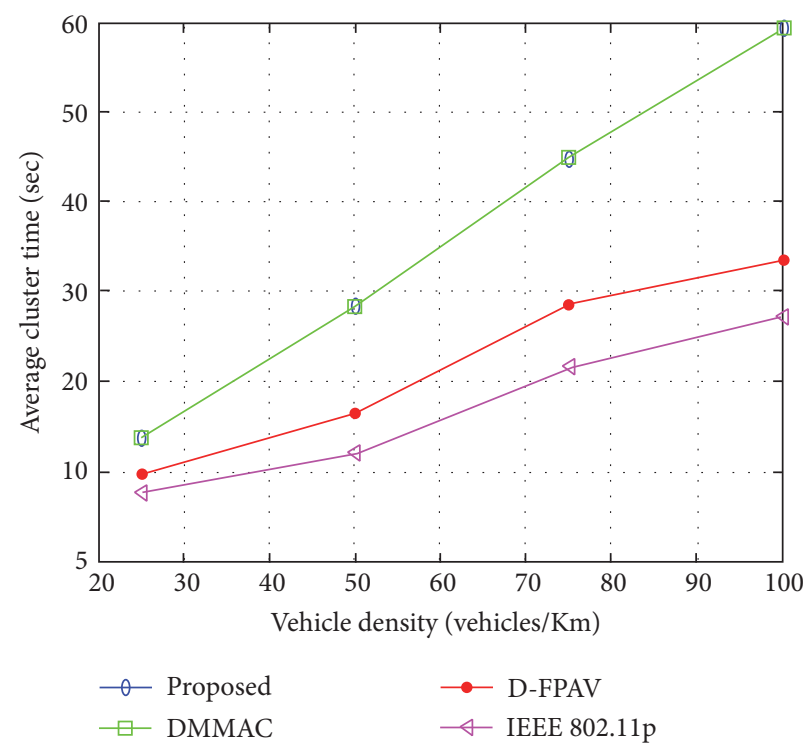

(b) $R=500$

Figure 12: Average CH time versus vehicle density for transmission range of (a) $300 \mathrm{~m}$ and (b) $500 \mathrm{~m}$.

concurrent transmission without compromising the reliability of any individual application seems far from reality under the prevailing MAC architecture. QoS requirements should be met for all concerning metrics and not merely specific ones.

(3) An integrated protocol accounting to single-hop and multihop broadcast needs to be designed that would dynamically schedule the messages based upon their priority level.
(4) The applicability of location independent services should be studied. Clustering requirements in QoS for nonsafety applications need to be explored.

(5) Estimating the level of beacon congestion and its control algorithms must focus on link and network status. Moreover, under majority of the scenarios congestion detection is not possible without sharing frequent information among vehicles, which can further alleviate congestion. 


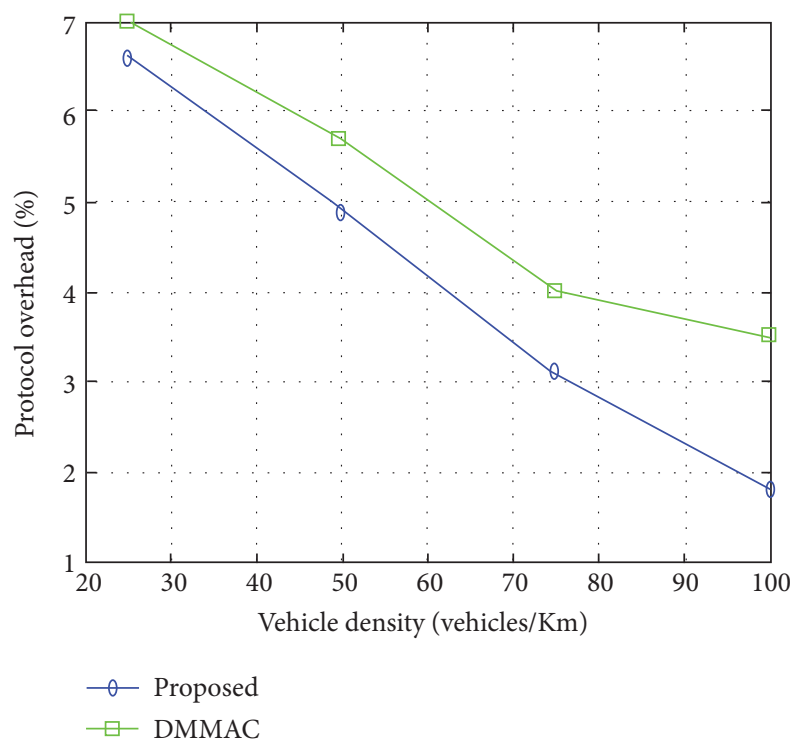

FIGURE 13: Cluster maintenance overhead versus vehicle density for $R=300 \mathrm{~m}$.

\section{Competing Interests}

The authors of this manuscript declare that there is no conflict of interests regarding the publication of this paper.

\section{References}

[1] IEEE Draft Standard for Information Technology-Telecommunications and information exchange between systemsLocal and metropolitan area networks-Specific requirements-Part 11: Wireless LAN Medium Access Control (MAC) and Physical Layer (PHY) specifications, Amendment 6: Wireless Access in Vehicular Environments, IEEE Std. 802.11p, July 2010.

[2] X. Ma, J. Zhang, X. Yin, and K. S. Trivedi, "Design and analysis of a robust broadcast scheme for VANET safety-related services," IEEE Transactions on Vehicular Technology, vol. 61, no. 1, pp. 46-61, 2012.

[3] M. Amadeo, C. Campolo, and A. Molinaro, "Enhancing IEEE 802.11p/WAVE to provide infotainment applications in VANETs," Ad Hoc Networks, vol. 10, no. 2, pp. 253-269, 2012.

[4] D.-J. Deng, C.-H. Ke, H.-H. Chen, and Y.-M. Huang, "Contention window optimization for IEEE 802.11 DCF access control," IEEE Transactions on Wireless Communications, vol. 7, no. 12, pp. 5129-5135, 2008.

[5] X. Yin, X. Ma, and K. S. Trivedi, "MAC and application level performance evaluation of beacon message dissemination in DSRC safety communication," Performance Evaluation, vol. 71, pp. 1-24, 2014.

[6] J. A. Sanguesa, M. Fogue, P. Garrido et al., "RTAD: a real-time adaptive dissemination system for VANETs," Computer Communications, vol. 60, pp. 53-70, 2015.

[7] R. S. Schwartz, A. E. Ohazulike, C. Sommer, H. Scholten, F. Dressler, and P. Havinga, "On the applicability of fair and adaptive data dissemination in traffic information systems," Ad Hoc Networks, vol. 13, pp. 428-443, 2014.
[8] S. H. Bouk, G. Kim, S. H. Ahmed, and D. Kim, "Hybrid adaptive beaconing in vehicular ad hoc networks: a survey," International Journal of Distributed Sensor Networks, vol. 11, no. 5, Article ID 390360, 2015.

[9] J. Liu and Q. Wang, "Position prediction based frequency control of beacons in vehicular Ad hoc networks," International Journal of Distributed Sensor Networks, vol. 11, no. 8, Article ID 631415, 2015.

[10] S. Najafzadeh, N. Ithnin, S. A. Razak, and R. Karimi, "BSM: broadcasting of safety messages in vehicular ad hoc networks," Arabian Journal for Science and Engineering, vol. 39, no. 2, pp. 777-782, 2014.

[11] V. D. Khairnar and S. N. Pradhan, "Simulation based evaluation of highway road scenario between DSRC/802.11p MAC protocol and STDMA for vehicle-to-vehicle communication," Journal of Transportation Technologies, vol. 3, no. 1, pp. 88-104, 2013.

[12] C. Chrysostomou, C. Djouvas, and L. Lambrinos, "Applying adaptive QoS-aware medium access control in priority-based vehicular ad hoc networks," in Proceedings of the 16th IEEE Symposium on Computers and Communications (ISCC '11), pp. 741-747, Kerkyra (Corfu), Greece, July 2011.

[13] Y. Allouche and M. Segal, "Cluster-based beaconing process for VANET," Vehicular Communications, vol. 2, no. 2, pp. 80-94, 2015.

[14] "IEEE Standard for Wireless Access in Vehicular Environments (WAVE) Multichannel Operation, Revision of IEEE Std 1609.4-2006," 2010, http://ieeexplore.ieee.org/servlet/opac? punumber $=5712767$.

[15] N. Gupta, A. Prakash, and R. Tripathi, "Medium access control protocols for safety applications in Vehicular Ad-Hoc Network: a classification and comprehensive survey," Vehicular Communications, vol. 2, no. 4, pp. 223-237, 2015.

[16] C.-T. Lu, C.-L. Chang, I.-H. Liu, and J.-S. Li, "Novel advanced broadcast scheme for efficient safety message collision alleviation in vehicular networks," Wireless Personal Communications, vol. 77, no. 4, pp. 2793-2816, 2014.

[17] K. A. Hafeez, L. Zhao, J. W. Mark, X. Shen, and Z. Niu, "Distributed multichannel and mobility-aware cluster-based MAC protocol for vehicular Ad Hoc Networks," IEEE Transactions on Vehicular Technology, vol. 62, no. 8, pp. 3886-3902, 2013.

[18] J. Sahoo, E. H.-K. Wu, P. K. Sahu, and M. Gerla, "Congestioncontrolled-coordinator-based mac for safety-critical message transmission in VANETs," IEEE Transactions on Intelligent Transportation Systems, vol. 14, no. 3, pp. 1423-1437, 2013.

[19] S. Bai, J. Oh, and J.-I. Jung, "Context awareness beacon scheduling scheme for congestion control in vehicle to vehicle safety communication," Ad Hoc Networks, vol. 11, no. 7, pp. 2049-2058, 2013.

[20] M. Azizian, S. Cherkaoui, and A. S. Hafid, "A distributed cluster based transmission scheduling in VANET," in Proceedings of the IEEE International Conference on Communications (ICC '16), pp. 1-6, IEEE, Kuala Lumpur, Malaysia, May 2016.

[21] H. A. Omar, W. Zhuang, A. Abdrabou, and L. Li, "Performance evaluation of VeMAC supporting safety applications in vehicular networks," IEEE Transactions on Emerging Topics in Computing, vol. 1, no. 1, pp. 69-83, 2013.

[22] H. R. Arkian, R. E. Atani, A. Pourkhalili, and S. Kamali, "Cluster-based traffic information generalization in Vehicular Ad-hoc Networks," Vehicular Communications, vol. 1, no. 4, pp. 197-207, 2014.

[23] K. Z. Ghafoor, J. Lloret, K. A. Bakar, A. S. Sadiq, and S. A. B. Mussa, "Beaconing approaches in vehicular Ad hoc networks: 
a survey," Wireless Personal Communications, vol. 73, no. 3, pp. 885-912, 2013.

[24] D31: European ITS Communication Architecture, "Overall Framework Proof of Concept Implementation," COMeSafety Consortium, Munich Germany, COMeSafety European Specific Support Action Public Deliverable, December 2009.

[25] J. J. Haas and Y. C. Hu, "Communication requirements for crash avoidance," in Proceedings of the 7th ACM International Workshop on VehiculAr InterNETworking (VANET '10), pp. 1-10, Chicago, Ill, USA, September 2010.

[26] P. Singh, R. Pal, and N. Gupta, "Clustering based single-hop and multi-hop message dissemination evaluation under varying data rate in vehicular ad-hoc network," in Advanced Computing and Communication Technologies: Proceedings of the 9th ICACCT, 2015, vol. 452 of Advances in Intelligent Systems and Computing, pp. 359-367, Springer, Berlin, Germany, 2016.

[27] X. Zhou and A. Boukerche, "An efficient adaptive MAC frame aggregation scheme in delay tolerant sensor networks," in Proceedings of the IEEE Global Communications Conference (GLOBECOM '14), pp. 277-282, Austin, Tex, USA, December 2014.

[28] J. Cho, A. S. Uluagac, J. Copeland, and Y. Chang, "Efficient safety message forwarding using multi-channels in low density VANETs," in Proceedings of the IEEE Global Communications Conference (GLOBECOM '14), pp. 70-75, December 2014.

[29] H. I. Abbasi, R. C. Voicu, J. A. Copeland, and Y. Chang, "Performance optimization of a contention based broadcasting algorithm in VANETs," in Proceedings of the IEEE Global Communications Conference (GLOBECOM '15), pp. 1-7, San Diego, Calif, USA, December 2015.

[30] F. Calì, M. Conti, and E. Gregori, "Dynamic tuning of the IEEE 802.11 protocol to achieve a theoretical throughput limit," IEEE/ACM Transactions on Networking (ToN), vol. 8, no. 6, pp. 785-799, 2000.

[31] J. Lloret, A. Canovas, A. Catalá, and M. Garcia, "Group-based protocol and mobility model for VANETs to offer internet access," Journal of Network and Computer Applications, vol. 36, no. 3, pp. 1027-1038, 2013.

[32] J. Zhou and K. Mitchell, "A scalable delay based analytical framework for CSMA/CA wireless mesh networks," Computer Networks, vol. 54, no. 2, pp. 304-318, 2010.

[33] The Network Simulator-ns-2, http://www.isi.edu/nsnam/ns/.

[34] F. Chiti, R. Fantacci, and G. Rigazzi, "A mobility driven joint clustering and relay selection for IEEE 802.11p/WAVE vehicular networks," in Proceedings of the 1st IEEE International Conference on Communications (ICC '14), pp. 348-353, Sydney, Australia, June 2014.

[35] http://dmf.unicatt.it/ pollini/sumo_user.shtml.

[36] M. Torrent-Moreno, J. Mittag, P. Santi, and H. Hartenstein, "Vehicle-to-vehicle communication: fair transmit power control for safety-critical information," IEEE Transactions on Vehicular Technology, vol. 58, no. 7, pp. 3684-3703, 2009.

[37] The CAMP Vehicle Safety Communications Consortium, "Vehicle safety communications project-task 3 final reportidentify intelligent vehicle safety applications enabled by DSRC," Tech. Rep. DOT HS 809 859, National Highway Traffic Safety Administration, US Department of Transportation, Washington, DC, USA, 2005. 


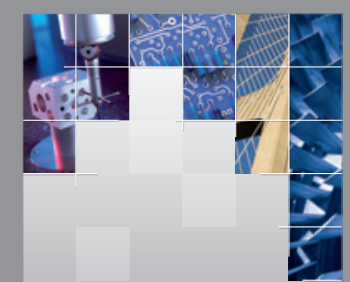

\section{Enfincering}
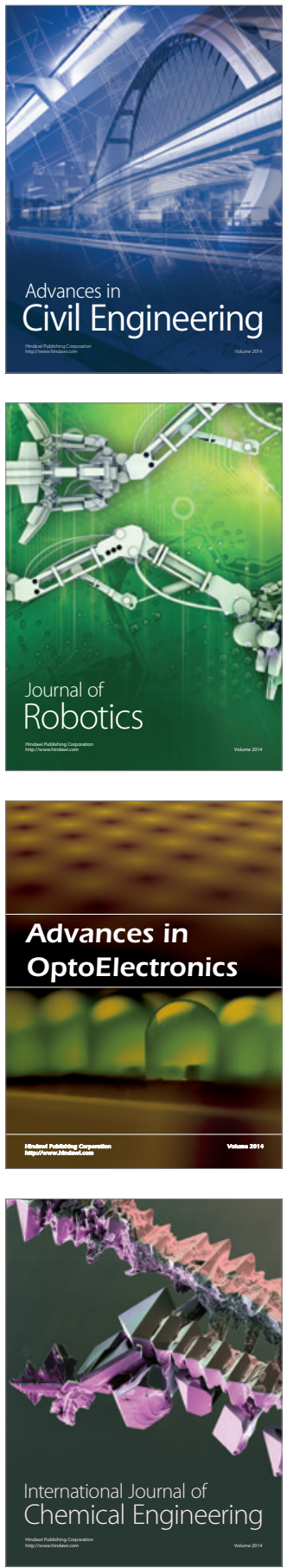

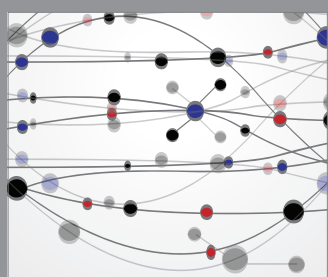

The Scientific World Journal

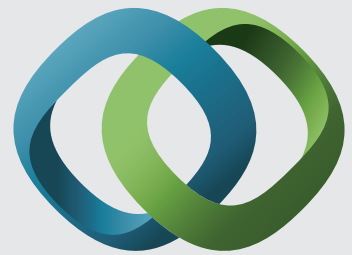

\section{Hindawi}

Submit your manuscripts at

https://www.hindawi.com
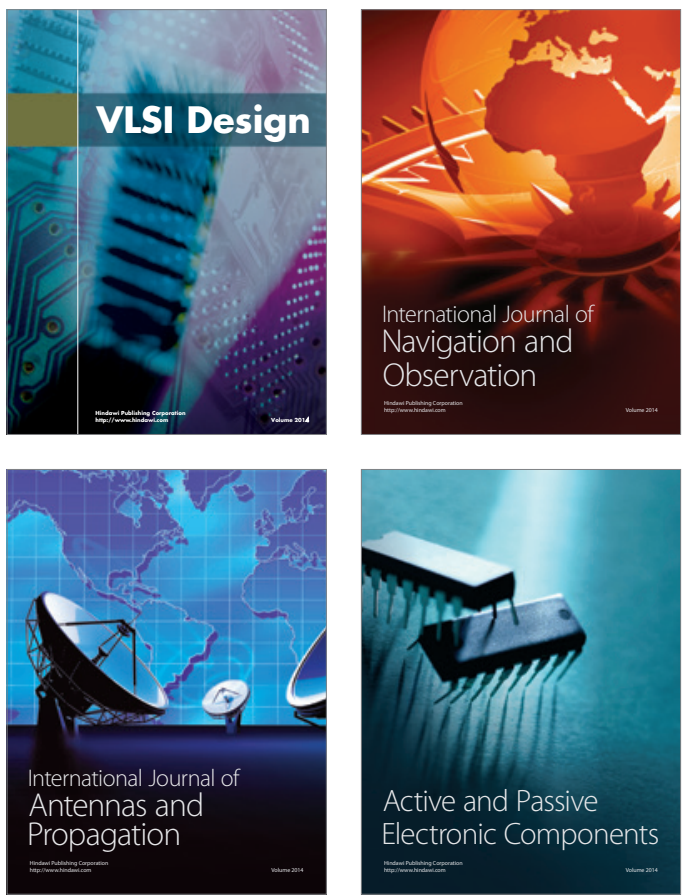
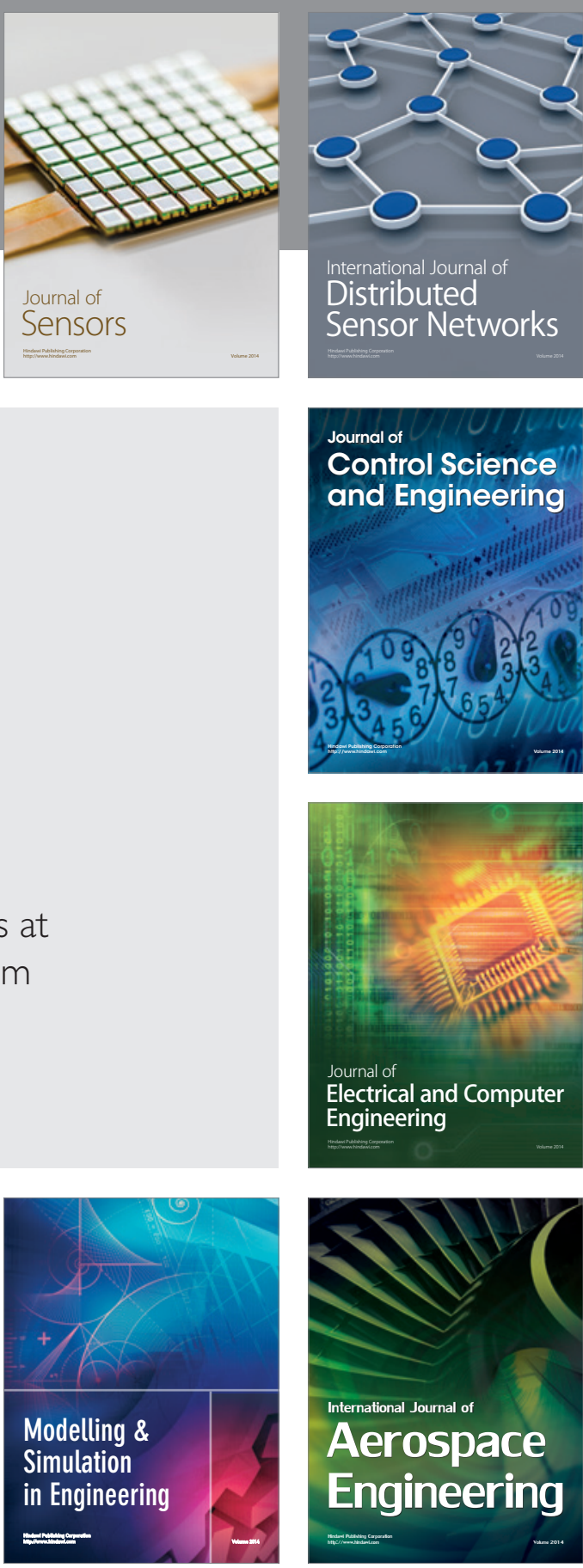

International Journal of

Distributed

Sensor Networks

$-$

Joumal of

Control Science

and Engineering
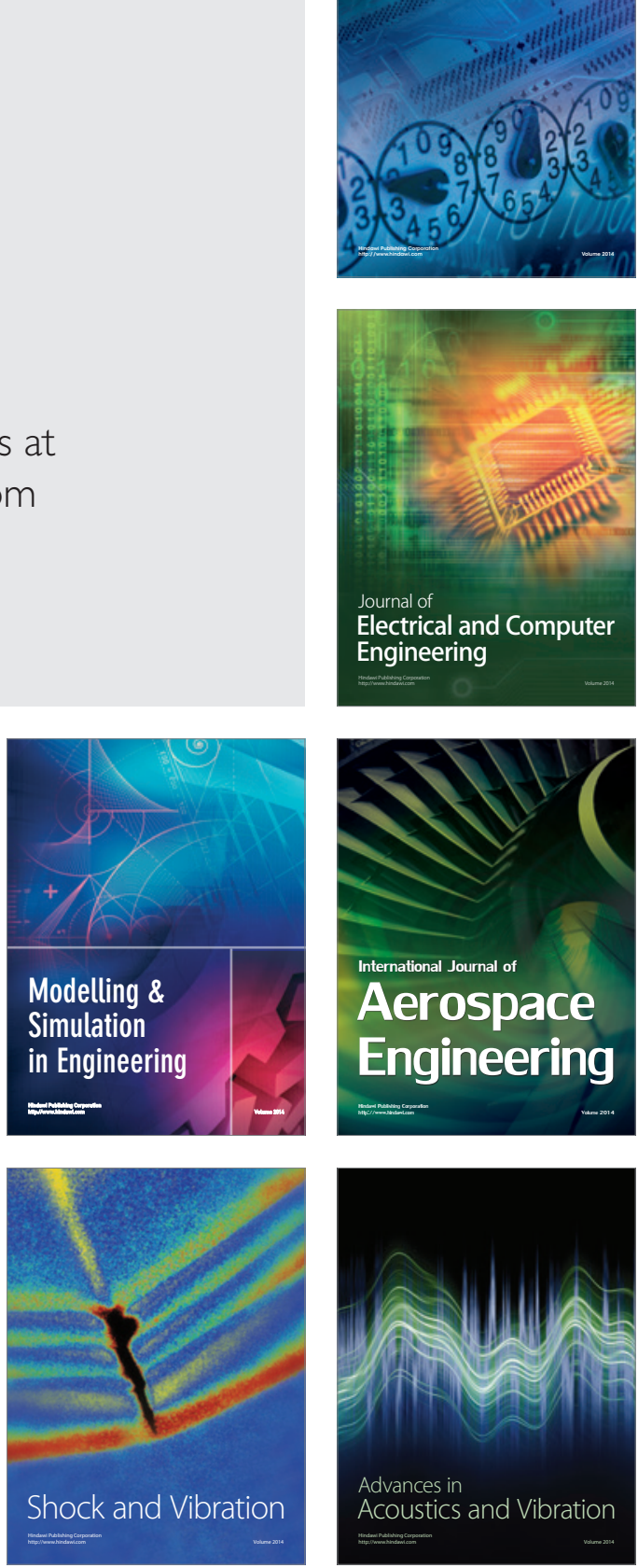